\title{
Stolpersteine bei der Praxisübernahme
}

\author{
Patrick Stacha, Fabian Teichmann ${ }^{\text {b }}$

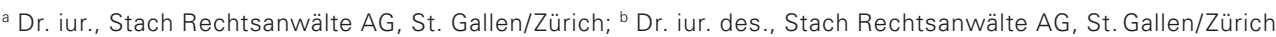

\section{Einleitung}

Praxisübernahmen sind ein zunehmend komplexes Thema. Beim Kauf und der Veräusserung von bestehenden Arztpraxen müssen verschiedene juristische Stolpersteine beachtet werden, um langwierige und kostenintensive Auseinandersetzungen zu vermeiden. Vorab gilt es zu bedenken, dass die Übernahme einer Praxis nahezu immer mit der Übernahme von Arbeitnehmern, Patienten(daten) und Honorarforderungen verbunden ist. Ferner müssen die Pflichten des Verkäufers, wie zum Beispiel die Einführung des Käufers in die Praxis und Gewährleistungsansprüche, geregelt werden.

\section{Vertragsgegenstand}

Zunächst ist der Vertragsgegenstand genau zu definieren. Hierbei ist zwischen der Übernahme eines Einzelunternehmens und derjenigen einer juristischen Person zu unterscheiden. Während beim Erwerb einer juristischen Person häufig nur die zu übernehmende Aktiengesellschaft oder Gesellschaft mit beschränkter Haftung bezeichnet werden muss, sind bei der Übernahme eines Einzelunternehmens sämtliche zu erwerbende Gegenstände einzeln aufzuführen. Das bedeutet in der Praxis, dass zumindest die Übernahme des Patientenstamms, der Geräte, des Inventars, der Telefonnummern, der Domain der Webseite, der Arbeitnehmer, des Mietvertrages und der Versicherungen aufgeführt werden müssen. Hierbei sind möglichst präzise Angaben zu machen, um spätere Streitigkeiten zu vermeiden.

Einen Spezialfall stellt der Eintritt in eine (oder Austritt aus einer) Gemeinschaftspraxis dar. In dieser Konstellation muss nicht nur der Wille des Käufers und des Verkäufers übereinstimmen, sondern es müssen regelmässig auch die anderen Ärzte der Praxis zustimmen. Ferner übernimmt der Käufer in diesen Fällen die Verträge nicht, sondern tritt in bestehende Vertragsverhältnisse zwischen mehreren Parteien ein. Je nach Umfang der Beteiligung an der Gemeinschaftspraxis, ergeben sich zudem unterschiedliche gesellschaftsrechtliche Konsequenzen. Man denke hierbei beispielsweise an den Schutz von Aktionärsminderheiten.

\section{Difficultés lors de la reprise d'un cabinet médical}

Le présent article se penche sur les différents aspects juridiques d'une reprise de cabinet médical, et plus particulièrement sur la teneur du contrat, la reprise des salariés et de la patientèle, la cession de créance d'honoraires, la mise au cou rant de l'acheteur et la garantie du vendeur. Les questions relatives à l'interdiction de la concurrence, aux assurances et à la planification fiscale sont également brièvement abordées.

\section{Übernahme von Arbeitnehmern}

Zunächst ist zu bedenken, dass der Arzt, der eine eigene Praxis führt, auch Unternehmer ist. Damit sind insbesondere im Hinblick auf allfällige Angestellte verschiedene Punkte zu beachten. Wird ein Betrieb oder Betriebsteil verkauft oder sonst übertragen, so gehen gemäss Art. 333 OR die Arbeitsverhältnisse automatisch auf den Erwerber des Betriebs über, sofern der Arbeitnehmer dies nicht ablehnt. Lehnt der Arbeitnehmer den Übergang ab, kommen die gesetzlichen, nicht die vertraglichen Kündigungsfristen zur Anwendung. Der Erwerber des Betriebes haftet für die Verbindlichkeiten des alten Arbeitgebers aus den Arbeitsverhältnissen vor dem Betriebsübergang mit. Das Bundesgericht hat letzthin entschieden, dass der frühere Arbeitgeber für Verbindlichkeiten nach dem Betriebsübergang noch bis zu demjenigen Zeitpunkt mithaftet, auf welchen die Arbeitsverhältnisse ordentlich gekündigt werden könnten [1].

\section{Übernahme von Patienten}

Neben Arbeitnehmern werden im Rahmen des Praxisverkaufs auch Patienten übernommen. Für diese wird gemäss Art. 4 Abs. 5 des Bundesgesetzes über den Datenschutz grundsätzlich eine ausdrückliche Einwilligung der betroffenen Patienten benötigt. Daher empfiehlt es sich, die Zustimmung zur Weitergabe von Patientendaten bereits bei der Aufnahme von (neuen) Patienten in einem Standardformular zu regeln. Sollte diese Einwilligung nicht vorliegen, sind zumindest alle Patienten über die Praxisübernahme in geeigneter Form zu informieren. Ihnen ist die Gelegenheit zu 


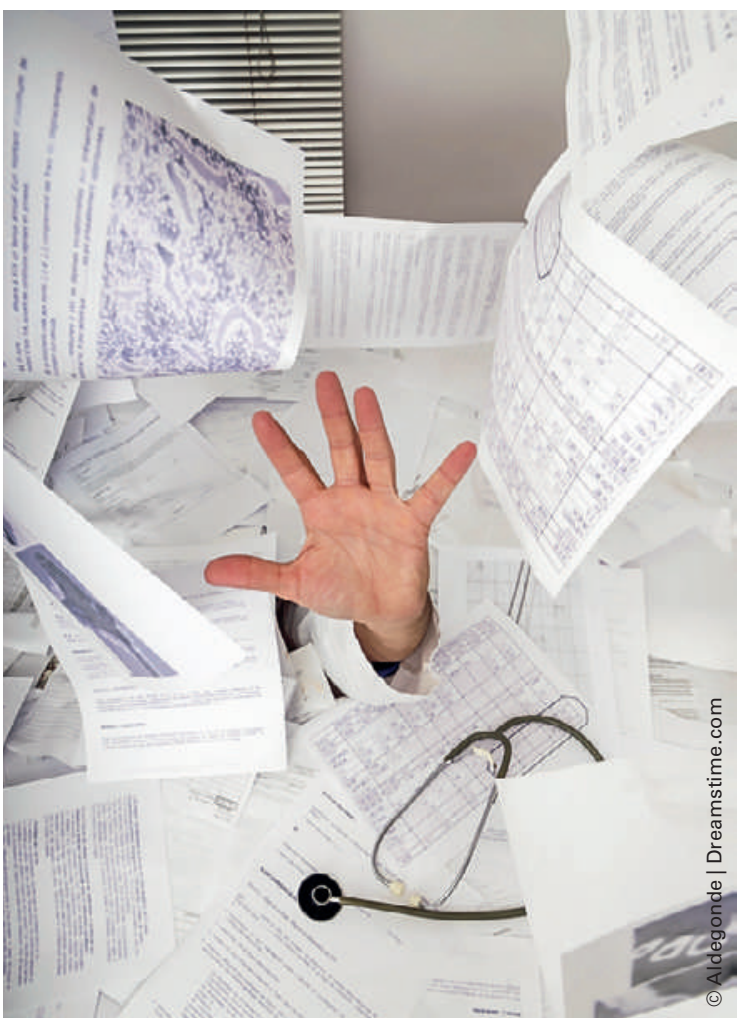

Damit man nach einer Praxisübernahme nicht im Chaos versinkt, gilt es vorab einiges zu bedenken: Nahezu immer ist mit der Übernahme von Arbeitnehmern, Patienten(daten) und Honorarforderungen zu rechnen. Und es gibt noch viele weitere juristische Stolpersteine.

geben, innert nützlicher Frist ihre Patientenakte abzuholen. Ferner sollte sich der Käufer verpflichten, die Patientendossiers des Verkäufers zu übernehmen und zehn Jahre lang aufzubewahren. Damit wird der Verkäufer von seiner Aufbewahrungspflicht befreit und die Praxisübernahme massgeblich erleichtert. In diesem Zusammenhang ist das Zwei-Schrank-Prinzip zu empfehlen, sofern nicht alle Patienten informiert werden konnten. Dabei werden die Dossiers von den Patienten, welche nicht informiert wurden, in einem separaten Schrank aufbewahrt. Idealerweise übernimmt der Nachfolger zum Geheimniskreis seines Vorgängers gehörende Mitarbeiter, welche bei Bedarf auf die entsprechenden Akten zugreifen können und dürfen.

Gleichzeitig gilt es zu bedenken, dass die oben ausgeführten Schritte nicht nur vor datenschutzrechtlichen, sondern auch vor strafrechtlichen Folgen schützen. Zu erinnern ist dabei an das gestützt auf Art. 321 StGB stipulierte Arztgeheimnis. $\mathrm{Zu}$ beachten ist, dass das Arztgeheimnis grundsätzlich auch gegenüber Ärztekollegen und Personen des Gesundheitswesens gilt. Eine Weitergabe von Patientendaten ohne Zustimmung der betroffenen Patienten könnte somit auch strafrechtliche Konsequenzen haben.

\section{Zession von Honorarforderungen}

Der Datenschutz spielt nicht nur bei der Übernahme von Patienten, sondern auch bei der Zession von Honorarforderungen eine entscheidende Rolle. Hierbei gibt es zwei Möglichkeiten. Entweder der übergebende Arzt rechnet seine bisherigen Behandlungen selbst $a b$, oder es erfolgt eine Zession von Honorarforderungen im Rahmen der Praxisübernahme. Eine Zession von Honorarforderungen führt zwangsläufig auch zu einer Preisgabe der Namen der Patienten. Damit ist unter Umständen auch eine (teilweise) Preisgabe der Krankengeschichte des Patienten verbunden. Dies ist insbesondere dann der Fall, wenn erbrachte Leistungen Rückschlüsse auf den Gesundheitszustand der Patienten zulassen. Eine Zession sollte deshalb im Hinblick auf das Arztgeheimnis nur mit Zustimmung der betroffenen Patienten erfolgen. Eine entsprechende Klausel könnte in einem Standardformular eingebaut werden, welches von allen (neuen) Patienten unterschrieben wird. Sofern keine Einwilligung des Patienten vorliegt, benötigt der Arzt für die Geltendmachung der Forderung unter Umständen eine Entbindung vom Berufsgeheimnis durch die vorgesetzte Behörde.

\section{Einführung des Käufers in die Praxis}

Wünscht der Käufer eine Einführung in die Praxis durch den Verkäufer, so empfiehlt es sich, Art und Umfang schriftlich festzuhalten. Mündliche Zusicherungen sind zwar grundsätzlich ebenfalls verbindlich, aber regelmässig nur schwer zu beweisen. Um eine geordnete Praxisübergabe zu gewährleisten, sollte u.a. vereinbart werden, in welchem Zeitraum der Verkäufer zur Verfügung steht. Ferner stellt sich regelmässig die rechtliche Frage, ob er mit längeren Einführungen oder Urlaubsvertretungen u.U. nach der Veräusserung als Arbeitnehmer für die Praxis tätig wird.

\section{Gewährleistungen des Verkäufers}

Allfällige Gewährleistungsansprüche, welche über das gesetzlich vorgeschriebene Mass hinausgehen, sind schriftlich festzuhalten. Denkbar sind unter anderem Gewährleistungsansprüche für das Inventar oder den Patientenstamm. Bei Letzterem ist jedoch Vorsicht geboten, da ein gewisser Schwund an Patienten nach einer Praxisübernahme durchaus nicht ungewöhnlich ist und somit der aktuelle Stand des aktiven Patientenstamms am Stichtag der Praxisübernahme nur schwer überprüfbar ist. 


\section{Konkurrenzverbote}

Während Konkurrenzverbote in Arbeitsverträgen für angestellte Ärzte aufgrund des besonderen Vertrauensverhältnisses mit den Patienten nur in sehr eingeschränktem Umfang möglich sind, können Konkurrenzverbote bei Praxisübernahmen durchaus von Vorteil sein, um zu verhindern, dass der Arzt, welcher seine Praxis gewinnbringend veräussert hat, in der unmittelbaren Nachbarschaft eine neue Praxis eröffnet. Derartige Konkurrenzverbote sind zeitlich, örtlich und sachlich zu beschränken. Diese Beschränkungen dürfen nicht übermässig sein, da sie sonst herabgesetzt werden können. Eine maximale zeitliche Beschränkung auf drei bis fünf Jahre erscheint in den meisten Fällen angemessen zu sein. Die örtliche Beschränkung kann sich auf einen Notfallkreis oder eine grössere Stadt beziehen. Eine Ausweitung auf den gesamten deutschsprachigen Raum wäre hingegen übermässig, da dies einem Berufsverbot gleichkommen würde. Auch die sachliche Beschränkung drängt sich in den meisten Fällen auf und ergibt sich aus der bisherigen Tätigkeit des Arztes (bspw. Hausarzt, Gynäkologe etc.). Viele Standardverträge, unter anderem der FMH-Mustervertrag, sehen jedoch Ausnahmen für gelegentliche Stellvertretungen vor. Damit selbige keine Hintertüre für unerwünschte Tätigkeiten des Verkäufers öffnen oder selbiger noch im von ihm gewünschten Umfang tätig sein kann, ist es jedoch meistens unerlässlich, diese Musterklauseln individuell anzupassen.

Das Konkurrenzverbot sollte zudem grundsätzlich entgeltlich sein. Bei einem Pauschalpreis für die Übernahme einer Praxis ist sodann zu empfehlen, die Entschädigung für das Konkurrenzverbot explizit als Bestandteil des Pauschalpreises auszuweisen. Zudem sollte eine Konventionalstrafe pro Übertretungsfall vereinbart werden. Allenfalls besteht auch ein Recht des Käufers, den rechtmässigen Zustand herbeizuführen.

\section{Versicherungen}

Es muss beachtet werden, dass gewisse mit einer Arztpraxis verbundene Versicherungspolicen gemäss Art. 54 VVG bei einer Praxisübernahme automatisch auf den Käufer übergehen. Der Käufer kann den Übergang des Vertrages durch eine schriftliche Erklärung bis spätestens 30 Tage nach dem Kauf ablehnen. Voraussetzung hierfür ist jedoch, dass der neue Eigentümer der Praxis Kenntnis von sämtlichen Versicherungsverträgen hat. Diese sind also bei der Praxisübernahme offenzulegen.

\section{Steuerplanung}

Eine rechtzeitige juristische Planung kann helfen, Steuern zu sparen. Unter bestimmten Voraussetzungen kann eine Überführung einer Praxis in eine juristische Person mindestens fünf Jahre vor der Veräusserung helfen, die Steuerlast des Veräusserers zu optimieren. Dies gilt insbesondere dann, wenn eine Personengesellschaft auf eine juristische Person übertragen wird. Sofern dabei die bisher für die Einkommenssteuerpflicht massgeblichen Steuerwerte übernommen werden und die Steuerpflicht in der Schweiz fortbesteht, werden die stillen Reserven u.U. nicht besteuert. Dies setzt jedoch voraus, dass innerhalb von 5 Jahren nach der Übertragung keine Beteiligungsoder Mitgliedschaftsrechte zu einem über dem übertragenen steuerlichen Eigenkapital liegenden Preis veräussert werden [2]. Voraussetzung für die Steuerersparnis ist jedoch, dass keine indirekte Teilliquidation vorliegt und die Beteiligung nicht als Geschäftsvermögen qualifiziert wird [3].

\section{Reugeld}

Abschliessend sollte u.U. auch die Vereinbarung eines Reugeldes in Erwägung gezogen werden. Dies ist insbesondere deshalb sinnvoll, weil im Rahmen von Praxisübernahmen häufig Anzahlungen geleistet werden, welche sowohl dem Käufer als auch dem Verkäufer eine gewisse Sicherheit für den Zeitraum zwischen dem Vertragsabschluss («signing») und dem endgültigen Vollzug der Transaktion («closing») gewähren sollen. Wenn ein Reugeld vereinbart wurde, kann der Käufer gegen Zurücklassung der Anzahlung und der Verkäufer gegen Erstattung des doppelten Betrages vom Vertrag zurücktreten. Das Reugeld soll also beide Parteien von einem Rücktritt abhalten, um den reibungslosen Verlauf der Praxisübernahme zu gewährleisten.

\section{Fazit}

Zusammenfassend ist festzustellen, dass die Praxisübernahme ein durchaus komplexes Thema ist, welches einer rechtzeitigen Planung bedarf. Sofern die oben genannten Punkte rechtlich klar geregelt werden, steht einer reibungslosen Transaktion nichts mehr im Wege.

\section{Literatur}

1 BGE 129 III 335

2 Art. 19 Abs. 1 Bst. B i.V.m. Art. 19 Abs. 2 DBG; Art. 8 Abs. 3 Bst. b i.V.m. Art. 8 Abs. 3 bis StHG.

3 Art. 2Oa Abs.1 Bst. a DBG; Art. 7a Abs. 1 Bst. a StHG; Art. 18 DBG, Art. 7 Abs. 1 i.V.m. Art. 8 StHG. 